\title{
The Impact of Covid-19 on Socio-Economic Change and Psychological Health of Moroccan Citizens
}

\author{
JIBRAILI Zineb, Research professor in management sciences, ENCGM, Cadi Ayyad University, \\ Research structure: LAREGO, Marrakech, Morocco \\ ABYRE Asmaa, Research professor in management sciences, FEG BM, Sultane Moulay Sliman University, \\ Research structure: LERSEG, Beni Mellal, Morocco \\ ANOUAR Hajar, Research professor in management sciences, ENCGBM, Sultane Moulay Sliman University, \\ Research structure: LERSEG, Beni Mellal, Morocco \\ JIBRAILI Malak, PHD student, ENCGC, Research structure LRPFG, Casablanca, Morocco
}

\begin{abstract}
The spread of coronavirus has an impact on citizens of the world, on both mental and financial sides. Indeed, citizens of the world are confined for weeks, this situation causes the increase in the state of anxiety and stress. This health crisis caused job losses, increased unemployment and therefore the appearance of financial and economic difficulties. In this perspective, we decided to conduct this study, the aim of which is to present the factors that contribute to increase in the level of anxiety and how it can be impacted by financial and economic level of Moroccan citizens. In this sense, we examined the effect of covid-19 on financial situation and level of distress of Moroccan citizens during the crisis period. We designed a survey to study firstly the determinants of social anxiety: personality, religious beliefs and level of exposure concerning COVID-19, and secondly, the impact of financial and economic situation of individuals during this period on their psychological state.
\end{abstract}

Keywords:- Covid-19, Social Anxiety, personality, exposure, religious beliefs, economic, Moroccan citizens.

\section{INTRODUCTION}

Coronaviruses are a large family of viruses that can cause disease in animals and some have the ability to be transmitted between animals and humans, a so-called "spillover event". In humans, several coronaviruses are known to cause respiratory infections ranging from the common cold to more serious illnesses such as Middle East Respiratory Syndrome (MERS) and Severe Acute Respiratory Syndrome (SARS). The most recently discovered coronavirus causes COVID-19 disease.

This infectious disease was first discovered in China, in the city of Wuhan in December 2019, and has spread globally. The World Health Organization (WHO) has called the Covid-19 outbreak a pandemic because of the severity of the spread of the virus worldwide. As of April 30,2020 , more than 3.25 million cases had been reported in 185 countries and territories resulting in more than 232,000 deaths compared to 1.01 people recovered.
In Morocco, 4423 confirmed cases have been registered in the different regions of the Kingdom, of which more than $50 \%$ of the cases concern the regions of Casa Safi (1120 cases) and Marrakech Safi (957 cases). Morocco has also recorded a total of 170 deaths due to the coronavirus pandemic, compared to 984 cures.

The new coronavirus COVID-19 can be transmitted from person to person. Symptoms include cough, fever, shortness of breath, muscle aches, sore throat or unexplained loss of taste or smell. Most people infected with the COVID-19 virus recover without requiring special treatment. The elderly and those with underlying medical conditions such as cardiovascular disease, diabetes, chronic respiratory disease and cancer are more likely to develop serious illness. There is no vaccine against coronavirus yet. Prevention consists of frequent hand washing, coughing into the crook of the elbow, keeping a distance of at least one metre, putting on a bib when going out and staying home to avoid any risk of contamination and only going out in an emergency.

The spread of coronavirus has an impact on the citizens of the world, on the mental and financial sides. Indeed, the citizens of the world are confined for weeks; this situation causes the increase in the state of anxiety and stress. This health crisis caused job losses, increased unemployment and therefore the appearance of financial and economic difficulties to individuals. In this perspective, we decided to conduct this study, the aim of which is to present the factors that contribute to the increase in the level of anxiety and how it can impact the financial and economic level of Moroccan citizens. Therefore, we ask four hypothesis: More the person is optimistic, less he becomes anxious; more the person is religious, less he becomes anxious; more the person is exposed to the covid19, more he becomes anxious and more the financial and economic situation of persons deteriorates, more he becomes anxious.

In this sense, we present in the next point social anxiety, its determinants and the relationship that may be linked between this psychological situation and the economic situation of Moroccan citizens. 


\section{LITERATURE REVIEW}

\section{$>$ Determinants of increasing the social anxiety during the covid-19 period}

Threaded, confined, obsessed by the fear of the unknown, humanity has never seemed so helpless in the face of a health threat such as that linked to the Coronavirus. Needless to say, pandemics have always had a negative impact on the psychological state of individuals ${ }^{1}$. Beyond the apparent symptoms (cough, respiratory problems, headaches, fever, etc.), Covid-19 - just like the former pandemic viruses - contribute to the development of unprecedented psychosocial anxiety. All this leads, as Glassner, (1999) pointed out, to the establishment of a real "culture of fear"2 in today's society.

Indeed, studies conducted by (Bai Y, \& al. 2014), (Cava MA \& al., 2005) have expressed citizens' fear of their own health or that of infecting others, during this period and were more likely to fear infecting family members than those who were not quarantined.

Not surprisingly, anxiety is a measure of distress that has been associated with a large number of traumatic events (Alvarez and Hunt 2005; Marshall et al. 2007). As an event like Covid-19 progresses, the public becomes more and more aware of the event taking place. This awareness may be due to either an exposure or an indirect exposure, such as the media. In addition, these indirect exposures can affect anxiety levels as much or more than actual exposure levels (Griffin, Dunwoody and Zabala 1998; Schlenger et al. 2002). It is therefore important to understand the various factors that contribute to an increased state of anxiety in a society during periods of an epidemic.

The irruption and subversive spread of the coronavirus in various modern societies has had as its main consequence the emergence of a feeling of increased social anxiety. It should be noted that there are several anxiety factors related to Covid-19 both globally and in Moroccan society. In the next point, we will present two factors that deal with the state of social anxiety during the epidemic period.

\section{- Exposure}

Many factors have an important role in the increased anxiety of people. Actually, it has been proven that exposure to traumatic situations are the source of chronic stress. Several studies, conducted in different contexts, have confirmed the positive relationship between the level of exposure and the worsening of the state of distress. They have proven that the people which are positioned in the risk zone, have an increasing degree of anxiety. This is a consequence of the anxiety provoked, thinking that they will be the next victim. This cognitive mechanism, as confirmed by Marshall \& al. (2007), associates with the model of "disaster", where distress is all the more powerful when one approaches the centre of the disaster. Likewise,
Fischhoff \& al. (2003) found that the proximity of the World Trade Centre was predictive of future terrorist risk judgments. Furthermore, Xiao-Fei Xie \& al., (2011), have shown that exposure to the phobogenic stimulus almost invariably elicits an immediate anxiety response. These authors confirmed, through their studies, that people in Chinese cities most affected by the SARS pandemic had anxiety disorders.

As a result, the literature has shown that social phobia (or social anxiety) would be characterized by clinically significant anxiety caused by exposure to a certain type of social or performance situation, often leading to behaviour avoidance.

However, others researchers have shown that there is not always a positive relationship between exposure to a pandemic and anxiety. As a matter of fact, the SARS epidemic, this infectious agent that have appeared in November 2002 in Guangdong province, China, has caused similar states of anxiety in different areas of the affected countries, even those that are not directly touched. The latter were notified by indirect channels, mainly by the media. As far as, we present, the study conducted by Schlenger \& al. (2002), who analysed citizens' reactions to the terrorist attacks of September 11 in America. These authors found that, among the factors studied (including direct exposure to the attacks), the number of hours of television watched during the days of this disaster, was the only significant predictor of general distress. In the same way, Bleich, Gelkopf and Solomon ${ }^{3}$, who measured the impact of the proximity factor on the increase in the state of anxiety in individuals, found that the level of exposure (operationalized as actual exposure to terrorist attacks and as vulnerable to terrorist attacks due to objective characteristics such as living in an urban setting vs. a rural location) was not related to post-traumatic stress disorder or other distress measures. They hypothesized that this lack of relationship was due to "the ubiquitous traumatic reality in Israel," which has been spread in part by media coverage.

In a second level, some research has shown that people who have been exposed to a well-defined risk are less psychologically affected than those who have not experienced it, but just imagined it. The fear of being affected and the danger increased in these people did not affect. Thus, we can present the study by Maderthaner $\&$ al. (1978) who found that participants living very close to a nuclear reactor assessed the risk of living near a nuclear reactor as being lower than that of participants living at an intermediate distance. Likewise, Halpern-Felsher et al. (2001) found that people who have experienced a natural hazard perceive the associated risk as less than people who have not been exposed to the hazard. 


\section{- Personality}

A second factor which has emerged from the literature and which positively impacts the rise of the level of social anxiety relates to the personality of the person. Some react more strongly or aggressively to negative events than others (Sjöberg 2000 In Stone \& Al., 2011). (X.-F. Xie \& al., 2011), in their study related to the impact of the spread of the SARS epidemic on the psychological state of citizens has shown that the state of anxiety is positively impacted by the internal control of the event. Which depends on the personality of the person.

Indeed, a person with an extroverted type A behaviour will have a different assessment of the situation and not the same reaction as a person with a type $B$ behaviour reflecting a rather introverted personality. For this study, we would like to deal with the extent to which the personality of our respondents affects their psychological state, by measuring their optimistic or pessimistic tendency. To do this, according to the study of (X.-F. Xie \& al., 2011), we will use the LOT life orientation test method of Scheier, (1994).

\section{$>$ The impact of Economic and financial situation of} individuals during covid-19 on their social anxiety state

In this period of health crisis due to the spread of the COVID-19 coronavirus pandemic, we are witnessing a recession in the world economy, including that of Morocco. According to the Prime Minister, more than $60 \%$ of SMEs stopped their activity. This obviously has a negative impact on the financial and economic situation of citizens (Reuters, 2020.) Many have lost their jobs due to stagnant activity in different industries. According to Petric, (2020), this represents an important risk factor for the development of mental health problems in addition to extensive measures of social distancing and quarantine. These people are now the most vulnerable group in terms of mental and financial health.

Psychological health can be affected by financial stress. Since money is an integral part of our lives, worrying about it all the time can lead to serious long-term psychological symptoms. According to Manly, (2019), When you're stressed about your finances, you can become very anxious, even depressed. People who have major concerns about their personal finances can become preoccupied, distracted, worried, dissatisfied, or even depressed. Overly stressed, a person is more likely to sleep less, be less focused, be more anxious and perform less well, which can negatively affect job performance. Under stress, blood flow and electrical activity decreases in the frontal lobe and prefrontal cortex, but increases in the parts of the brain that regulate survival functions, such as the amygdala, Forbes, (2020). Since these parts of the brain contribute to skills such as problem solving, concentration, planning and impulse control, malfunctioning of these areas can cause us to make poor decisions. When we worry or panic because of our financial situation, our decisions become more impulsive: they are motivated by the need to survive. "We act quickly and decisively, but not always with foresight. Since money is an integral part of our lives, worrying about it all the time can lead to serious psychological symptoms in the long run. "When you're stressed out about your finances, you can become very anxious, even depressed". This state of stress and anxiety leads to a blockage and then to a loss of productivity (individual efficiency) and a lack of motivation which can further aggravate the individual's financial situation.

\section{MATERIALS}

The appearance and spread of Covid-19 have caused discomfort in various countries around the world, causing mental and financial suffering. In this perspective, we decided to explore the relationships between these two variables, while identifying and studying the main factors responsible for the levels of distress experienced, namely personality attributes, religious beliefs and level of exposure. As the first step, in order to contextualize the model, we interviewed six Moroccan experts. the analysis of these results made it possible to conclude from the need to add a third determinant of anxiety, relating to religious beliefs. Its indicators were obtained following the analysis of certain numbers of writings in different contexts. As the second step, we adopted a quantitative study, in this sense, we randomly interviewed Moroccan citizens from different areas of the kingdom. Data was collected by using socials media mainly by sending emails and sharing the survey on Facebook. In the end, we received 705 responses.

We have processed the data on SPSS. To present the relationship between the level of exposure and the state of anxiety, we calculated the variable load scores, then we analysed it by using the ACP technical. Then, we proceeded to study the model by structural equations 'PLS'. In this sense, we proceeded to the exploratory then confirmatory study.

\section{$>$ Exposure}

We asked questions about the gender, age, education, occupation, and their cities. In addition, we asked about participants' exposure to Covid-19. We used 6 items to evaluate that attribute, degage from many studies, Marshall et al. (2007), Xiao-Fei Xie et al., (2011). These questions were: 'Have any Covid-19 patients been found around you?' and 'Have any suspicious cases been found around you?' Participants answered 'yes' or 'no' to that items. An exploratory analysis conducted on pls allowed us to eliminate four items whose value is less than 0.7. In the end, two indicators were chosen EXPO4 $=0.95$ and EXPO5 $=0.97$ with a Cronbach's alpha $=0.85$.

\section{$>$ Religious beliefs}

The questionnaire asked for six items regarding the religious beliefs of the participants and its capacity to control the state of anxiety which increases during this period of health crisis and confinement. The examples of questions are "'my belief in Allah will not disappoint me 'and' I feel protected from the virus when I make my prayers'. Participants rated how each of these statements described themselves on a seven-point scale, with' $1=$ strongly disagree, $5=$ totally agree". An exploratory 
analysis conducted on pls allowed us to eliminate four items whose value is less than 0.7. In the end, two indicators were chosen RELI3 $=0.55$ and RELI4 $=0.98$ with a Cronbach's alpha $=0.85$.

\section{Personality attributes}

We measured participants 'optimism and pessimism tendencies via the 'Sensation Inventory' (Scheier, Carver, and Bridges 1994). The questionnaire is made up of 8 items, for example, 'I always look on the bright side of things' and 'I seldom expect everything will go smoothly in my future.' Participants rated how each of these statements described themselves on a five-point scale, with' $1=$ strongly disagree, $5=$ totally agree". An exploratory analysis conducted on pls allowed us to eliminate five items whose value is less than 0.7 . In the end, three indicators were chosen PERS5 $=0.76$, PER6=0,71 and PERS7 $=0.91$ with a Cronbach's alpha $=0.79$.

\section{Anxiety}

Anxiety has been measured based on several studies. The scale consists of 10 items, for example, 'I am depressed because of confinement', and 'Coronavirus spread stresses me' Participants rated how each of these statements described themselves on a five-point scale, with' $1=$ strongly disagree, $5=$ totally agree". An exploratory analysis conducted on pls allowed us to eliminate seven items whose value is less than 0.7. In the end, three indicators were chosen ANX4 $=0.85, \mathrm{ANX} 7=0,71$ and ANX8 $=0.85$ with a Cronbach's alpha $=0.75$.

\section{Economy}

Economy has been measured based on several studies. The scale consists of 6 items, for example, 'the idea of going out to work stresses me', 'you have a stable job', 'you lost your job because of covid-19'. Participants rated how each of these statements described themselves on a five-point scale, with' 1 = strongly disagree, 5 = totally agree". An exploratory analysis conducted on pls allowed us to eliminate three items whose value is less than 0.7. In the end, three indicators were chosen ECON2 $=0.85$,
ECON3=0,81 and ECON4 $=0.81$ with a Cronbach's alpha $=0.777$.

\section{RESULTS}

\section{A. Effects of exposure to Covid-19 on state anxiety}

As the first step of this analyse, we targeted the citizens of the 11 zones of Morocco in order to cover the entire territory. The main purpose of this was to increase the credibility of this study. In this sense, among the 706 participants in this survey, 150 people $(21.24 \%)$ reported that patients with COVID-19 had been found around them, $250(31.12 \%)$ confirmed that they do not know of any cases affected by those around them. However, 98 (14\%) claimed that suspected cases of Covid-19 had been found around them and ultimately $208(29.50 \%)$ said that they didn't know of any suspicious cases on their area.

As a complement to this first analysis, we conducted a second one, which consisted in testing whether the level of exposure of the participants in Covid-19 affected their state of anxiety. In this sense, we calculated the scores of the two variables, then we analysed them on SPSS by using principal component analysis.

In this analysis, we looked at the location of the respondents to our questionnaire. In this perspective, we assumed that the proximity of quarantine zones would have an increased effect on the deterioration of people's psychological health. Thus, we classified the respondents according to their location. This distribution was made respecting the geographical distribution of the Moroccan kingdom, composed of the following regions: Dakhla Oued Dahab, Laâyoune Sakia El Hamra, Souss Massa, Beni Mellal Khenifra, Oriental, Rabat salé Kenitra, Saraa tafilalet, Fès meknes, Tanger Tetouan, Marrakech Safi and Casa settat. So, in the end, we had 11 distinct groups of participants.

Then, we calculated the scores for the two variables "Anxiety" and "Exposure" by referring on geographical area. The results of the PCA are shown in Figure 1 below. 
ISSN No:-2456-2165

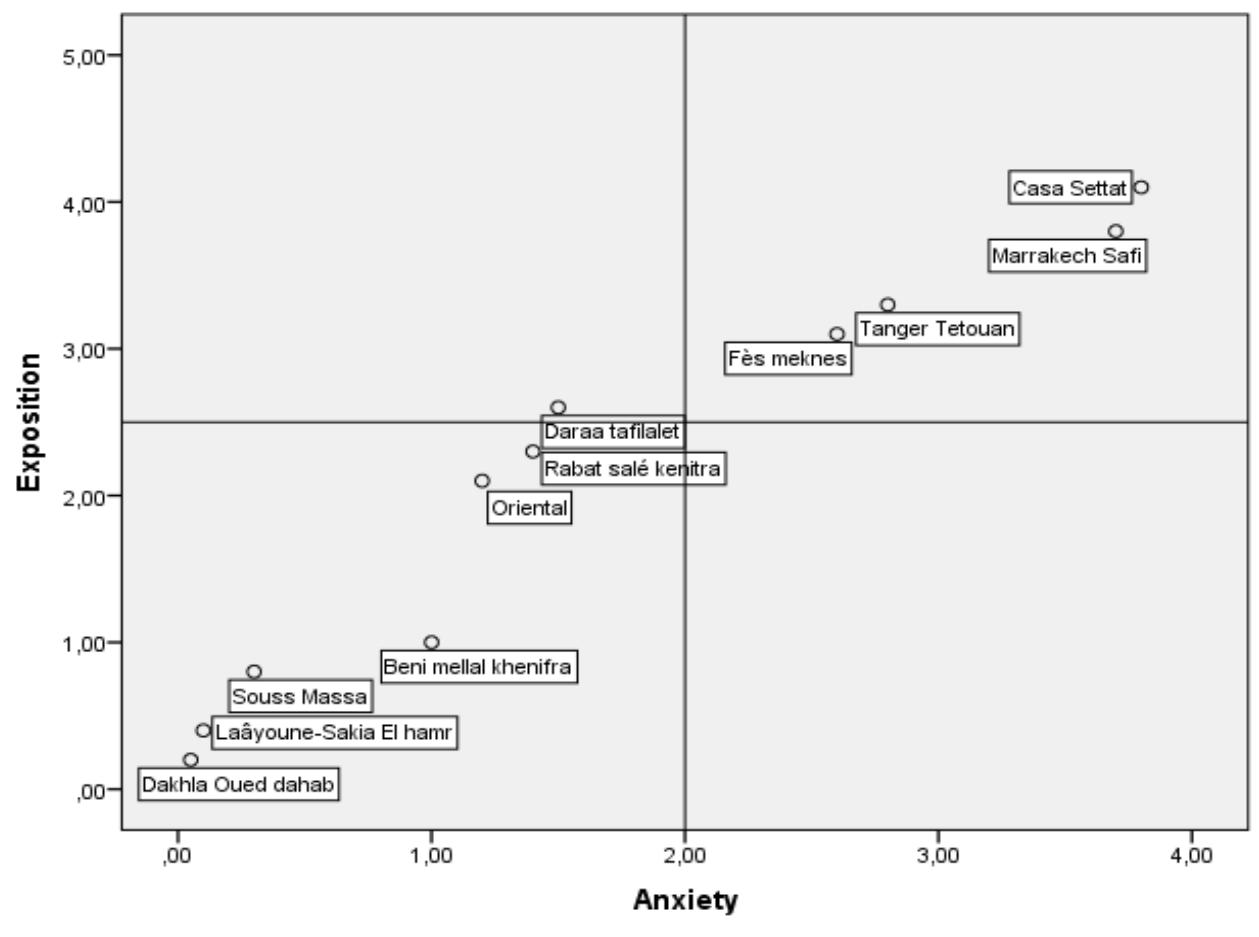

Fig 1

As shown in Figure 1, people living in the areas most affected by the Covid-19 pandemic are more exposed to increasing of their state anxiety. As we can notice, the citizens of the two regions most affected by this virus: "Casa-Settat" and "Marrakech Safi" have mental disorders, and this since the appearance of the virus. However, the people who live in the least affected areas: Dakhla oued dahab, Laâyoune sakia el hamra don't feel any anxiety.

\section{Summary:}

The results of the analysis of the objective characteristics associated with Covid-19 claim that more the person is exposed to the risk situation presented by the spread of the pandemic, more the level of the anxiety increases. At this level, it is important to examine the impact of other factors on the evolution of distress, and how this psychological state could impact the economic level of citizens during the spread of this pandemic.

\section{B. Impact of economic situation on state anxiety of Moroccan citizens during the Covid-19 period}

The results of the first study, which consisted in analysing the objective characteristics associated with Covid-19, claimed that more the person is exposed to the risk situation presented by the spread of the pandemic, more he becomes anxious. At this point, it is important to examine the impact of other factors on the evolution of the level of distress, and how this psychological state could impact the economic level of citizens during the spread of this epidemic.
The general structure of the model (see Figure 2) present that personality attributes, religious beliefs and level of exposure would influence anxiety and the change on financial and economic situation of citizens can increase their anxiety state.

\section{Model specification}

We formed two models to test the hypotheses. The following aspects have been taken into account in the specification of the model. Firstly, the model 1 include all of the potential links that seemed reasonable given our previous research on this subject. (Xiao-Fei Xie, 2011) showed in his work that exposure to the SARS epidemic and personality dimensions can have direct and positive influence on people's anxiety levels. Similarly, we added a third variable, released from the first step, relating to the contextualization study. Interviews with Moroccan experts on that area prompted us to add religious beliefs as the third determinant of social anxiety. Thus, we will study its direct and negative influence on the level of anxiety, more the person is a believer and the less he is exposed to an increase in his state of anxiety.

The second model tests the impact of the change in the financial and economic situation of Moroccan citizens on their psychological dimension. These five variables were measured by items released from the literature and the exploratory study. 


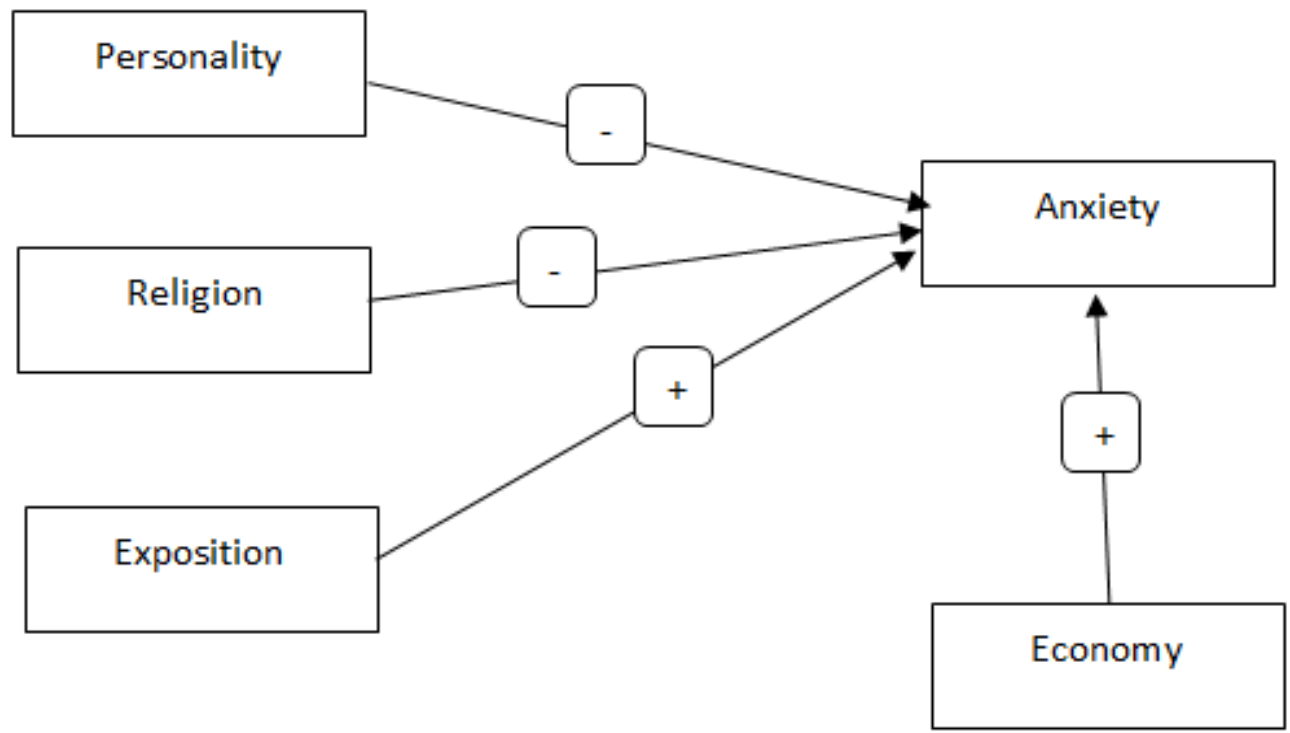

Fig 2

\section{Statistical analysis}

We focus on testing the relationships between the variables in our model in order to validate or refute each of the hypotheses. In this perspective, we will go through two stages: the first relates to the explanation and adjustment of the measurement model through three tests: reliability, convergent and discriminant validity. The second will consist first of all in evaluating the overall validity of the model and then in testing the hypotheses of the structural model. We will use SmartPLS 2.0 software for this purpose.

\section{- Analysis of the measurement model}

The measurement model, also called an external model, represents the assumed linear relationships between the latent variables and the manifest variables. To get it, we will have to follow the following three steps:

- Reliability of items

- Convergent validity

- Discriminant validity

\section{$\checkmark$ Convergent validity}

The convergent validity calculated on the basis of the average variance shared between a variable and its items (Hulland, 1999). Researchers who used PLS used internal consistency, developed by Fornell \& Larcker, (1981) and adopted the instructions proposed by Nunnally, (1994), who considers the threshold of 0.7 as a record for "modest" compound reliability (composite reliability).

\begin{tabular}{|c|c|}
\hline Variables & Composite Reliability \\
\hline Anxiety & 0,858104 \\
\hline Economy & 0,867596 \\
\hline Exposition & 0,985623 \\
\hline Personality & 0,902421 \\
\hline Religion & 0,765317 \\
\hline
\end{tabular}

Table 1

The values of convergent validity are acceptable. Indeed, the constructs relating to exposure and personality have a strong internal coherence with a composite reliability that exceeds 0.9 .

The other constructs have good internal consistency with values greater than 0.8 .

\section{$\checkmark$ Discriminant validity}

Discriminant validity represents the traditional methodological complement of convergent validity. It consists in proving that the item is linked more strongly to its variable (AVE) than to the other constructs in the model. (Mourre, 2013). 
ISSN No:-2456-2165

\begin{tabular}{|c|c|c|c|c|c|c|}
\hline & AVE & Anxiety & Economy & Exposition & Personality & Religion \\
\hline Anxiety & 0,769978 & 0,8774839 & & & & \\
\hline Economy & 0,886086 & 0,925925 & 0,94132141 & & & \\
\hline Exposition & 0,855647 & 0,736698 & 0,905925 & 0,92501189 & & \\
\hline Personality & 0,953856 & 0,820692 & 0,726572 & 0,793531 & 0,97665552 & \\
\hline Religion & 0,736767 & 0,793531 & 0,73475 & 0,726572 & 0,820692 & 0,85835133 \\
\hline
\end{tabular}

Table 2

As this table indicates, all the cross-contributions of the variables are less than the square root of the AVE, which implies the validation of the discriminant validity of our construct.

\section{- Structural model assessment and hypothesis testing}

By using PLS regression, the analysis of the results obtained allowed us to stabilize the measurement model. In the following, we will assess the overall validity of the model and then test the hypotheses of the structural model.

The overall quality of the model: the adequacy test (GoF)

The Goodness of fit index is a statistical test that determines the level with which the sample data correspond to a normal distribution of a population.

For our model, the GoF, which is calculated on the basis of the average of the different constructs of the explained variance $\left(\mathrm{R}^{2}\right)$, the redundancy index and the community, presents a satisfactory level, GoF $=$ root $((0$, $30) *(0.56))=0.42$. The threshold recommended in the literature being 0.30 , this reflects on the one hand, a good quality of the links between the measurement variables and the latent variables, and on the other hand, a good quality of the structural relationships.

After having evaluated the predictability of the model through the Goodness Fit index, we will proceed in this second step to testing the hypotheses. It consists first of all in examining the level of significance of the standardized coefficients (Path coefficient) of the relationships between the latent variables. A Bootstrap type simulation is carried out for this purpose. Thus, and in order to test the research hypotheses, we calculated the correlation coefficients between the variables (Path coefficient) under the SmartPLS software. The results are shown schematically in the figure below:

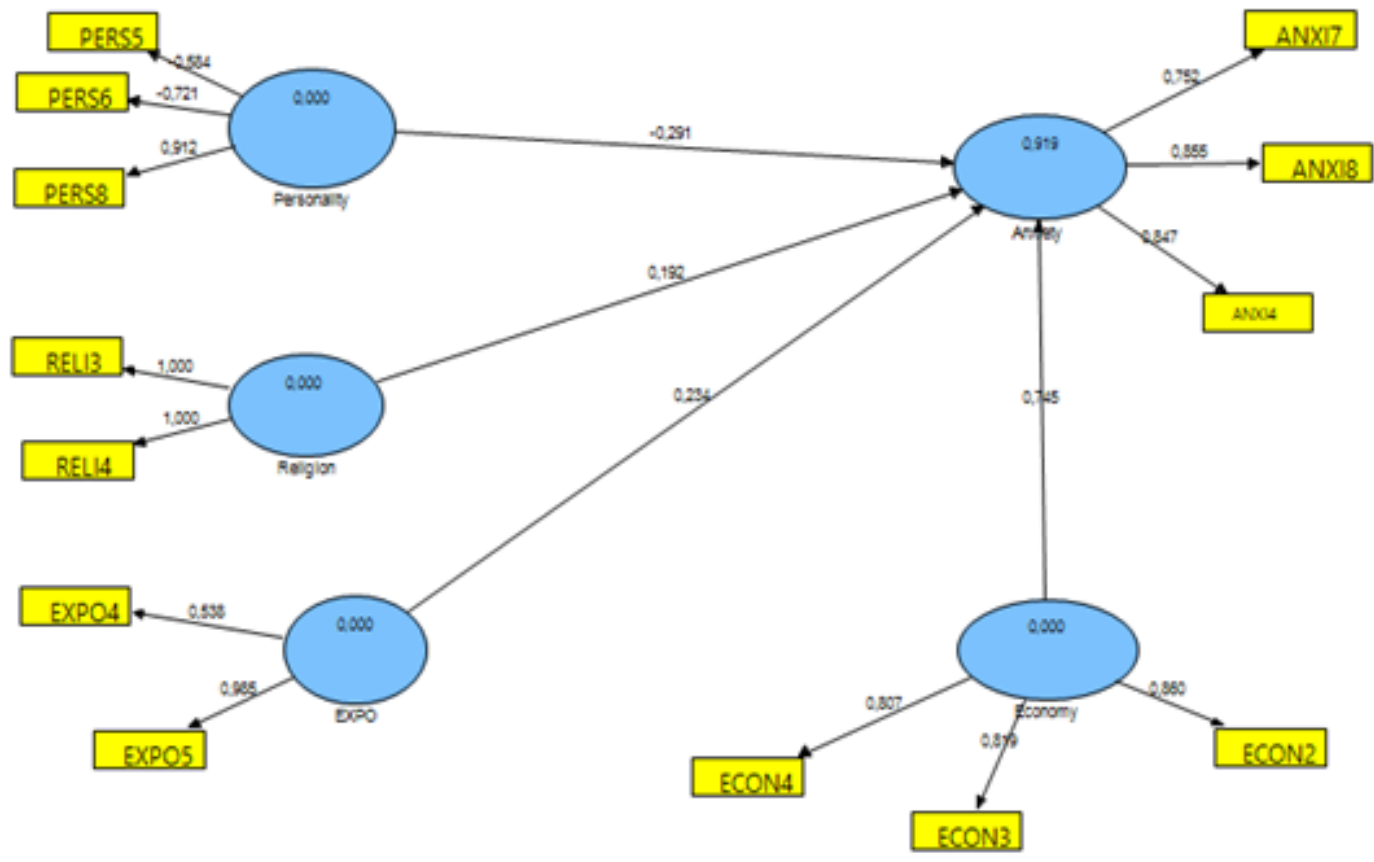

Fig 3

\section{Summary}

Determinants of social anxiety : As Figure 3 shows, the way participants reacted to Covid-19 influenced their level of anxiety. Indeed, the study has shown that the person's personality is a determining factor of the state of their social anxiety. More the person is optimistic, less he becomes anxious, with a Beta of -0.30 and a T-student of 7.18, which agrees with the conclusion of Xiao (2015). Likewise, the various analyses carried out have demonstrated the negative relationship between religious beliefs and anxiety. With a Beta of -0.13 and a T-student of 2.43 , the hypothesis tested, which states that more the 
person is religious, less he becomes anxious was validated. This result confirms the statements made by the respondents during the contextualization study.

In addition, the proximity of covid-19 also influences state of social anxiety, both directly and through the above variables. living in the most epidemic areas and therefore being more exposed to the risk of contamination has a positive effect on the increase in citizens' level of anxiety. This was confirmed by the statistical analyses carried out on SmartPLS.2, with a correlation level of 0.79 and a tStudent of 6.80 .

Economy and Social anxiety: The test of fourth hypothesis, which states that more the financial and economic situation of the person deteriorates, more he becomes anxious has been validated with a correlation coefficient of 0.92 and a t- student of 3.35. Thus, the strong association between the economic situation and the psychological state of the individual was also confirmed by this study, which agrees with the results of Manly, (2019).

\section{DISCUSSION}

The purpose of this document was threefold. Firstly, we tested the extent to which the level of exposure to the epidemic could have a negative impact on the person's psychological state, the analyses proved that being in a quarantine zone was a major determinant of the anxiety experienced.

Secondly, we examined determinants of anxiety state using structural equation modelling. Among these factors, the two most systematically related to distress were personality and religious beliefs. We found that more the person is optimistic and religious, less he becomes anxious.

As a third objective, we tested the relation that can be between the economic situation and psychological state of Moroccan citizen during the period of Covid-19. The various questions and analyses have led to the conclusion that this situation has negatively influenced the economic level of individuals. Some were dismissed for stopping their businesses; others had to quit their jobs outright, for fear of contamination. As a result, many of them have encountered financial difficulties. This state must have a negative influence on the psychological health of Moroccan citizens, through the rise in the level of anxiety about this new experience. This obviously joins studies conducted in different contexts including those of Manly, (2019) and Forbes, (2017) which have shown that more the person faces financial difficulties, more their state of anxiety increases.

We also found a greater fear of a recurrence of Covid19 in women than in men. Family responsibilities increase a person's level of fear and worry about an infectious disease outbreak.
At the end, the level of perceived risk associated with a disaster will be influenced by the level of awareness and knowledge of a person related to the disaster. Government programs aimed at raising such knowledge and awareness influence peoples' perceptions, and may help a society to become better prepared, and be more in control of a disaster situation; however, such programs may also have detrimental effects, as a result of raising people's anxiety levels. More studies are needed to understand the effect of this psychological and economic state of Moroccan citizens during the covid-19 period on the change in their consumption behaviour, then of the socio-economic situation of citizens during the post- Covid19.

\section{REFERENCES}

[1]. Alvarez, J., and M. Hunt. 2005. Risk and resilience in canine search and rescue handlers after 9/11. Journal of Traumatic Stress 18: 497-505.

[2]. Bai Y, Lin C-C, Lin C-Y, Chen J-Y, Chua C-M, Chou P. Survey of stress reactions among health care workers involved with the SARS outbreak. Psychiatry Serv 2004; 55: 1055-57.

[3]. Bults et al.: Perceived risk, anxiety, and behavioural responses of the general public during the early phase of the Influenza A (H1N1) pandemic in the Netherlands: results of three consecutive online surveys. BMC Public Health 2011 11:2

[4]. Bleich, A., M. Gelkopf, and Z. Solomon. 2003. Exposure to terrorism, stress-related mental health symptoms, and coping behaviors among a nationally representative sample in Israel. Journal of American Medical Association 290: 612-20.

[5]. Cava MA, Fay KE, Beanlands HJ, McCay EA, Wignall $R$. The experience of quarantine for individuals affected by SARS in Toronto. Public Health Nurs 2005; 22: 398-406.

[6]. Fischhoff, B., R.M. Gonzalez, D.A. Small, and J.S. Lerner. 2003. Judged terror risk and proximity to the World Trade Center. Journal of Risk and Uncertainty 26: 137-51.

[7]. Forbes D., 2020, What is COVID-19 doing to our mental health? First published on March 19, 2020 in Health \& Wellbeing.

[8]. Fornell, C.; Larcker, D.F. (1981). Evaluating Structural Equation Models with Unobservable Variables and Measurement Errors. journal of Marketing Research, 18, 39-50.

[9]. Glassner, B. (1999). The Culture of Fear: Why Americans Are Afraid of the Wrong Things.

[10]. Hulland, J. (1999). Use of Partial Least Squares (PLS) in strategic Management Research: Areview or four recent studies. Strategic Management Journal, 20, 195-204.

[11]. Maderthaner, R., G. Guttmann, E. Swaton, and H.J. Otway. 1978. Effect of distance upon risk perception. Journal of Applied Psychology 63: 380-2. 
[12]. Marshall, R.D., R.A. Bryant, L. Amsel, E.J. Suh, J.M. Cook, and Y. Neria. 2007. The psychology of ongoing threat: Relative risk appraisal, the September 11 attacks, and terrorism-related fears. American Psychologist 62: 304-16.

[13]. Manly, 2019, Create the Life of Your Dreams by Making Fear Your Friend

[14]. Mourre, M.L. (2013). La modélisation par équations structurelles basée sur la méthode PLS : une approche intéressante pour la recherche en marketing. Paris, France.

[15]. Reuters., 2020, COVID-19: Global economy already in recession. 20-03-2020

[16]. Petric, 2020, Negative mental health effects of covid 19 pandemic and panic

[17]. Scheier, M.F., C.S. Carver, and M.W. Bridges. 1994. Distinguishing optimism from neuroticism, Journal of Personality and Social Psychology 67: 1063-78.

[18]. Schlenger, W.E., \& Al., 2002. Psychological reactions to terrorist attacks: Findings from the national study of Americans' reactions to September 11. Journal of American Medical Association 288: 581-8.

[19]. Sjöberg, L. 2000. Factors in risk perception. Risk Analysis 20: 1-11.

[20]. X.-F. Xie \& al., 2011, The 'Typhoon Eye Effect': determinants of distress during the SARS epidemic, journal of risk research, 